\title{
THE CONCEPT OF FORM IN GEOMETRY: SOME CONSIDERATIONS CONCERNING SCIENCE AND MATHEMATICS EDUCATION
}

\author{
Paolo Bussotti, \\ University of Udine, Italy
}

The concept of form is one of the most intuitive within our experience. When we say that two objects of different dimensions have or do not have the same form there is not properly a reflexion behind this claim. Rather, it is, at all appearances, based on our visual faculties, which is perfectly in order in the context of our daily life. This intuitive and visual notion of form is suitable to the necessities of our practical, or also esthetical, experience. However, on second thought, things are not so easy: suppose that I look at an object and I find that it is circular. I claim, hence, that it is a circle and my statement is correct. Another person looks at this object from another point of view and sees that this object is an ellipsis or a hyperbola or a parabola. He is not wrong. This person is not the prey of a dream or of a hallucination. He is observing the world from another point of view, or as usually told in mathematics and physics, from another reference frame. A further example is even more indicative: it is well known that, if one treats the problem of the planetary orbits around the Sun as a two bodies problem, the orbit is an ellipsis: but, in respect to what reference frame is it an ellipsis? It is in respect to the barycentre of the system Sun-planet. Since such a barycentre lies, in our two-bodies hypothesis, within the Sun (but not, obviously, in its geometrical centre), one can say that, approximately, seen from the Sun, the orbit is an ellipsis. However, seen from the Earth, the orbit of a planet, for example of Mars, does not appear at all is an ellipsis: it is a strange figure with several loops and rather irregular. Then: what is the real form of Mars' orbit? The answer is that there is not such a "real form". It depends on the perspective. For most of the problems concerning astronomy, it is opportune to establish our point of view in the barycentre of the system Sun-planet, but this is not compulsory. At this point, one can feel discouraged: what is happening? Does no objectivity exist? Are maybe right the irrationalists? Is any experience similar to an esthetical experience? Is the objectivity of mathematics and physics taken away? The answers to all these questions are: "NO". Mathematics and physics have their kind of objectivity and they are the most and only suitable instruments we have to achieve a scientific knowledge of the universe. However, we have to renounce to a sort of absolute, of metaphysical concept of objectivity, and, in connection with this, of form. It is possible to define a form with precision, but we have to clarify the system, the "reference frame" within which we are operating. What is necessary to get objectivity is a transcription language which allows us to pass from a reference frame to another. At this point, I and all other persons will know that what I, from the Earth, see as a strange loop-figure, looks like an ellipsis when it is seen from the barycentre of the system Mars-Sun. The objectivity of the concept of form is modified into a higher kind of objectivity, which concerns the precise language transcribing the events seen from a certain system (point of view) into those seen from a different system.

Since science education has the duty to teach not only the techniques of mathematics and science, but, together and, in my opinion, before the techniques, the nature of mathematics and science as well as their conceptual foundations, it is necessary that the learners are instructed to reason within the general frame traced through the previous considerations. As always, a general frame is not sufficient for the ideas to be clear. Some examples are necessary. Because of this, in what follows some more concrete considerations on the concept of form in geometry will specify the preceding contents. These reflections are suitable for learners attending the last two years of a high school-lyceum. 
There is a mathematical discipline which, in some sense, is an exact transcription of our intuitive idea of form: it is the Euclidean geometry. First of all, it would be necessary to explain how the idea of geometrical figures as points, lines, surfaces and volumes, is born and what it means. For, it is clear that in our experience no mathematical line or surface exists. However, I give for granted that this problem has been death with and solved in a previous phase of education.

The Euclidean geometry is based on five axioms. They are:

1. A line can be drawn from a point to any other point.

2. A line (nowadays we say "a segment") can be extended indefinitely.

3. Given a centre and a radius, a circle can be drawn.

4. All right angles are equal.

5. If a line intersects two other lines so that the interior angles on one side of the intersecting line are less than two right angles, then the lines meet on that side. This is the famous Parallel Postulate. These axioms create the universe of the Euclidean geometry. Probably they derive from intuition, but once they are posed, all the properties of the Euclidean geometry must derive from these axioms and from their logical consequences. No other external intervention is permitted

Now, with regard to the geometrical form, how can it be defined? A possible and acceptable definition is: "the geometrical form of a figure is the information remaining when abstracting from its spatial position, its dimensions and its orientation". This means that, for example, to rotate or to reflect a figure in a mirror does not change its form.

If anything has to be deduced from the axioms and its logical consequences, the concept itself of form has. And, in fact, Euclid offers several interesting propositions concerning the forms of the figures existing in his geometry. In this respect, two very important propositions are the 16th and the 17th of the Elements' first book (in what follows, I will use notations as $I, 16, I, 17$ ) where Euclid proves respectively that in a triangle any external angle is greater than any not adjacent angle and that the sum of the internal angles of a triangle is equal or less than two right angles. While proving these propositions, Euclid uses only the axioms 1-4, not the fifth one. Therefore, forms as "a triangle in which the sum of the internal angles is bigger than two right angles" cannot exist in the Euclidean plane. This is not to be given for granted because, for example, the sum of the internal angles of any triangle drawn on a spherical surface is bigger than two right angles. Starting with proposition I, 29 Euclid begins to use the parallel postulate, to which he had not resorted in the initial 28 propositions. Something apparently strange happens: this postulate allows us to offer a precise delimitation of the Euclidean forms because it permits to specify not only that in a triangle any external angle is bigger than each of the two not-adjacent internal angles, but that it is exactly equal to their sum and that, consequently, the sum of the internal angles of a flat triangle is exactly equal to two right angles (Euclid I, 32). Therefore, the form "Euclidean triangle" has this property: "independently of the sides' dimensions, the sum of its internal angles is equal to two right angles". Since all the flat polygons can be obtained by decomposing them into triangles, proposition I, 32 offers a very strong condition on the form of the Euclidean figures. We also discover that the form is - at all appearances - connected to the fifth postulate. In a universe, in which the fifth postulate would not be valid, some strange things might happen to the forms! With regard to the concept of surface, all the Euclidean propositions concerning the surface of a flat polygon depend on I, 36, where it is stated that "Parallelograms posed on the same basis and between two parallel lines are equivalent". In the proof of this theorem, the parallel postulate is used. Since, by means of the infinitesimal methods developed in the course of history of mathematics, the surfaces and the volumes of the curved figures have been reduced to those of the rectilinear ones, this means that the Euclidean concept of area also depends on the fifth postulate. If we deny it, it is reasonable to guess that the concept itself of surface or volume of a figure has to be modified.

But, there is something more: probably the concept of similarity - jointly with that of parallelism - is the most important in the Euclidean geometry. In the fifth book of his Elements, Euclid introduces the concept of proportion and in the sixth book he defines when two polygons are similar: they are if: 1) they have the same number of sides and hence of angles; 2 ) their corresponding angles are equal; 3 ) they have the corresponding sides in direct proportion. Do pairs of such polygons exist? Well, in the fundamental proposition Vl, 18, Euclid shows how "to describe on a straight line a rectilinear figure which is similar to another similar and similarly posed rectilinear figure". Therefore, similar figures exist and are construable by rule and compass. The thing is that in the course of the proof, Euclid resorts to already mentioned proposition I, 32, where the parallel postulate is used. Thence, two observations are necessary: 
1) The concept of similarity captures our intuitive idea of what a form is. For, such an idea is based on the conviction that, given a figure, it is possible to construct a figure having the same shape, but different and arbitrary dimensions.

2) The concept of similarity depends, at least in the presentation given by Euclid (but, as we will see, the existence of non-Euclidean geometries shows that no theory of similarity can exist without the fifth postulate), on the parallel postulate. This means that this concept might dramatically change if such a postulate were modified.

All in all: in the Euclidean world, similar figures exist and the concept of form is established on the basis of the notion of similarity: two figures have the same form if and only if they are similar. In terms of geometrical transformations, the Euclidean geometry is the group of the similarities. It is easy to offer the equation of a similarity.

What kind of world, what kind of reference frame is that of the Euclidean geometry? The correct answer is that it is the extension to the infinity of our local visual experience. The term "local" is here important. In our visual experience we do not see parallel lines. Two lines, which are locally Euclidean parallel converge in our visual experience (the example of two railway tracks is emblematic), but with our intellectual assessment we interpret such situation as a distortionary effect imputable to our sight. Therefore, we think that the two lines will maintain their distance even at infinity. In this sense, the Euclidean geometry can be interpreted as the extension at infinity and idealization of our local visual experience. We feel that we are at rest in a point of the universe and presume that our local experience is universally valid. The Euclidean geometry formalises this situation.

Let us now suppose that we move from our place, for example along a straight line: we see that something changes. What before appeared to be, i.e., an equilateral triangle, now seems a triangle, but not equilateral; what appeared a square is still a quadrilateral, but not a square; what appeared a circle now seems an ellipsis or a parabola or a hyperbola and vice versa. These visual experiences are, hence, not described by the Euclidean geometry. It seems that a new and more general geometry is necessary. In particular, the concept of form, which appeared so well established by the similarities, becomes something different and more general. The similarities preserve the Euclidean form because they transform a triangle into a similar triangle, a quadrilateral into a similar quadrilateral, a circle into a circle, and so on. But now, when we see the things while changing our point of observation, the concept of form itself changes, because we see that the form "triangle" is preserved; the form "quadrilateral" is preserved, but not the form equilateral or right triangle or the form square or parallelogram. Is it possible to construct a geometry in which this new and more general concept of form is stated, namely a geometry where "triangle" is a form, but "equilateral" o"right triangle" is not, that is where the triangles (as well as the quadrilaterals, the pentagons, etc.) are treated as a sole equivalence class of figures, as a sole form? The answer is yes. By means of the appropriate steps, it is possible to show that such a geometry has to be based on a modification of the parallel postulate, so that it is "axiomatically" posed that all the lines mutually intersect. The Euclidean parallels will intersect at infinity, but, in fact, there will be no need to distinguish between Euclidean and not Euclidean parallels. All the lines will be treated in the same manner. From an intuitive point of view, this is comprehensible if one thinks that the parallel axioms are connected with the angular relations among three lines; but if the angular relations are not preserved in our new geometry, then the parallelism will not be preserved, as well. And if a property is not preserved by the fundamental transformations of a certain geometry, it cannot be a fundamental property of such a geometry. The branch of mathematics based on the above traced considerations is, of course, the projective geometry.

This induces considerations which are necessary within the context of science and mathematics education for the learners to achieve a clear idea of such a fundamental concept as that of form: the form is a relative concept. It depends on the axioms we pose at the basis of a certain geometry. The intuitive concept of form is, in fact, not so intuitive because, as we have seen, it changes according to our kind of experience. Once a series of axioms is established, the concept of form is defined by abstraction relying upon such axioms and their logical consequences. It is also appropriate to underline that while posing the axioms, we create a mathematical world with its rules and features: for example, it is easy to define a metric, a concept of distance with the Euclidean geometry because the similarities, though do not preserve the distances, preserve their ratios, whereas the projectivities do not preserve such a ratio. Therefore, while "distance" or, at least "ratio of 
distances" is a concept of the Euclidean geometry, it is not a concept of the projective geometry. Within this branch of mathematics, a concept, which, in a sense (said quite imprecisely) has a role comparable to that of ratio of distances in the Euclidean geometry exists, but its examination goes beyond the aim of this editorial.

However, a common conceptual and notational language exists which allows us to grasp if we are moving in a projective or in a Euclidean context because all the transformations can be transcribed into analyticalalgebraic equations. It is, hence, relatively easy to discover that the Euclidean transformations - and, thus, the similarities, too - are a subset of the projective transformations. This means that we pass from projective to Euclidean geometry when specifying some particular elements which in projective geometry are posed in general terms.

However, the concept of form can be generalised even more. For, the branch of mathematics called topology deals with transformations which are even more general than projectivities. They are the bi-continuous transformations. As in the case of Euclidean and projective geometry, a system of axioms has to be posed, on which basis the topological transformations are deduced and, thence, the new concept of form is defined. Since the transformations have to be bi-continuous, the only property which is preserved by them is the so called connection. From an intuitive point of view, this means that a figure with no "hole" is topologically equivalent to any figure with no hole, but it is different from a figure with one "hole"; which, in its turn, is different from a figure with two "holes", and so on. This means that figures as triangles, quadrilateral, squares, circles, and so on are all equivalent from a topological perspective. They belong to the same topological class. While figures as a circular crown or a quadrilateral with a hole belong to a different topological class of figures. Therefore, here another concept of form exists: it depends only on the kind of connection.

We have passed from the rich word of Euclidean geometry where there are many different classes of figures to the projective geometry where several classes of Euclidean figure converge into one class. Since in the projectivities the points of intersections are conserved, this means that all the triangles belong to the same class, all the quadrilateral to the same, and so on. Finally, in topology, the concept of form becomes so general that neither the intersection points are appropriate to denote classes of figures - that is forms -, but only the bi-continuous transformations are. Exactly because the world of topology is less rich from an ontological point of view in respect to that of the projective and of the Euclidean geometry, it allows us to deal with more general and profound properties of the figures and of the space itself and, hence, of the forms. Is the world of topology a completely abstract mathematical object or does it maintain a connection with an intuitive approach to the external world? Well, thought difficult, the answer seems to be that it has. Piaget and his School, also working with mathematicians as Dieudonné and Choquet, arrived at thinking that when children are around three years old, they reason "topologically" and not according to the canons of the Euclidean or the projective geometry (Piaget-Beth-Dieudonné-Lichnerowicz-Choquet-Gattegno, 1955; Bussotti, 2013). For, in the course of several experiments, children gathered in the same class figures as triangles, quadrilaterals, circles, etc; in another class figures with a "hole" and so on. Obviously, the interpretations of these experiments are not univocal, but an entire pedagogical School was born basing on the idea that - starting from elementary instruction - science and mathematics education should be based on a topological approach (Bussotti, 2013).

Projective geometry and topology are not in contradiction with Euclidean geometry, rather they are an extension of it. For example, if we specify where two lines cut and replace the concept of "straight lines which cut at infinity" with the equivalent one of "parallel lines", we regain the Euclidean geometry starting from projective geometry. The difference is in the generality of projective geometry, where a concept as that of distance does not exist (or, at least is definable through a series of not easy conceptual steps). In contrast to this, some geometries, where the concept of distance is defined, which, hence, are as specific as the Euclidean geometry, exist, which are based on the negation - not on a generalization - of the fifth postulate. They are the non-Euclidean geometries. Let us focus only on the so called hyperbolic geometry. In this case the fifth postulate is replaced by the following one: "given a straight line $r$ and a point $P$ external to it, two parallel lines to $r$ from P exist". All the other four postulates of the Euclidean geometry maintain their validity. This apparently little change in the fifth postulate has, in fact, enormous consequences on the forms and configurations of the hyperbolic geometry. Only to mention some of the most intuitively surprising, it is possible to prove that:

1) the angle of parallelism is not a right angle. It can be any acute angle depending on the distance between $r$ and $P$. The greater the distance, the smaller the angle of parallelism. This implies the very important fact that a connection between segments and angles exists in hyperbolic geometry. That is, any segment has its associate angle of parallelism. Therefore, a relation between the dimensions of the sides of the 
polygons and the amplitude of the angles exists, which is not the case in the Euclidean geometry. A further and fundamental implication is that no similar figures can exist unless they are isometric;

2) the distance between two parallel lines is not constant; it decreases while going far from the point $P$. Therefore two parallel lines are asymptotic. In the Euclidean geometry two straight lines cannot be asymptotic. Hence, the new form "asymptotic straight lines" is typical of the hyperbolic geometry;

3) hyper-parallel straight lines exist. Given the point $P$ and the line $r$, these are lines from $P$ which do not cut $r$, but whose distance from $r$ has a minimum different from 0 . This means that these lines do not cut $r$ and are not asymptotic to $r$. For any line $r$ and any external point $P$, there is an infinity of hyper-parallel straight lines to $r$ passing through $P$. No form of this kind exists in the Euclidean geometry;

4) the sum of the internal angles of a triangle is less than two right angles and it diminishes while increasing the dimensions of the sides. This confirms that two similar but not isometric figures cannot exist. For, if, i.e., we consider an equilateral triangle, the sum of its internal angles varies inversely as the dimensions of the sides. This means that the form "equilateral triangle" has a substantial modification in respect to Euclidean geometry insofar as the sum of the internal angles is variable and less than two right angles;

5) analogously the sum of the internal angles of a quadrilateral is less than four right angles and it becomes less while the dimensions of the sides increase. Therefore, a quadrilateral having equal the four sides and the four angles exists, but it does not maintain the feature of the Euclidean squares, in which all the angles are right and, hence, constant. Furthermore, no rectangle can exist. The form of quadrilateral is, thus, very different that in Euclidean geometry;

6) in Euclidean geometry, only two kind of bundles of lines in a plane exist: those of cutting lines and those of parallel lines. In the hyperbolic plane geometry, there are three forms with regard to the bundles of lines: the cutting lines, the parallel lines and the hyper-parallel lines. Given a point $P$, the set $S$ of points symmetric to $P$ in respect to a bundle of parallel lines is, in Euclidean geometry, a line perpendicular to those of the bundle and passing through $P$. In the hyperbolic geometry $S$ is a new figure, which does not exist in the Euclidean geometry. It is called horocycle. This line, perpendicular to all the lines of a bundle of parallel straight lines is not a straight line. It has a series of very important properties. Some of them are shared with the Euclidean straight line and some of them with the Euclidean circles. From an intuitive point of view, it might be considered as a circle whose centre is at infinity. The intuitive reason why the horocycle shares properties with the Euclidean straight lines is that they might be considered as circles whose centre is at infinity, too. The form "horocycle" is fundamental in hyperbolic geometry, but it does not exist in the Euclidean geometry.

Many other considerations might be added and other kinds of "strange" geometries might be considered: elliptical, spherical, non-Archimedean, non-Pythagorean geometry and so on. However, what explained is enough to grasp that the concept of form is not absolute, but relative to the geometrical environment in which we decide to operate. Such a decision depends on the axioms we establish. Any geometrical world has its specific forms. A transcription language offered by abstract and ordinary algebra exists which allows us to pass from a geometrical universe to another geometrical universe, regaining in this manner, the concept of "objectivity", but the meaning of this concept is now different from the meaning in our common sense or in the metaphysical thought. The considerations concerning the notion of geometrical forms seem to me necessary in mathematics and science education to introduce the learners within all the difficult and complex nuances of the relations between what is objective and what is subjective. And regard to our external world? The answer is that, with the relativity theory the connections among the different points of view of the observers, between the different reference frames are far more important than in the Newtonian physics, because, as well known, concepts as that of simultaneity have only a local validity. On the other hand, as it is the case of the different geometries, a language of transcription exists which allows an observer to know exactly how a different observer perceives the space-time relations. A new and more general kind of objectivity is gained. Therefore, while speaking of the relativity of the concept of form in geometry, the teacher will also introduce the learner to grasp several aspects and nuances of the relation objectivity-subjectivity, which will be extremely useful when the theory of relativity will be faced. Furthermore, since the non-Euclidean geometries also play a role in the general theory of relativity, the discussion of the concept of form is also useful in the perspective of the education in physics.

In this way, a solid and thorough educative itinerary can be traced for science and mathematics education. 
ISSN 1648-3898/Print/

ISSN 2538-7138/Online/

\section{References}

Bussotti, P. (2013). Vittorio Checcucci and his contributions to mathematics education. A historical overview. Problems of Education in the $21^{\text {st }}$ Century, 53, 22-39.

Piaget, J. - Beth, E.W. - Dieudonné, J. - Lichnerowicz, A. - Choquet, C. - Gattegno, C. (1955). L'Enseignement des Mathématiques [The teaching of mathematics]. Neuchatel and Paris: Delachaux and Niestlé. Italy.

E-mail: paolobussotti66@gmail.com

Website: http://www.scienzainrete.it/documenti/autori/paolobussotti 